\title{
THE LIFT OF A DELTA WING AT SUPERSONIC SPEEDS*
}

\author{
BY \\ H. J. STEWART \\ California Institute of Technology
}

1. Introduction. The use of the two dimensional linearized theory of supersonic flows in the solution of airfoil problems as introduced by Ackeret ${ }^{1}$ has been extremely successful in solving these problems and the results have generally been completely satisfactory for engineering purposes. The generalization of these results to the three dimensional finite span problems has, however, progressed rather slowly due to mathematical complications. The flow near the tip of a rectangular wing was given (incorrectly) by Schlichting. ${ }^{2}$ The drag of a "delta" wing (a wing having an isosceles triangle for its planform with the symmetric vertex pointing into the oncoming flow as in Fig. 1) has been determined by Puckett. ${ }^{3}$ These two flow patterns and many other technically interesting finite span flow problems are particular cases of conical flows. A conical flow is one for which the fluid properties (pressure, velocity, etc.)

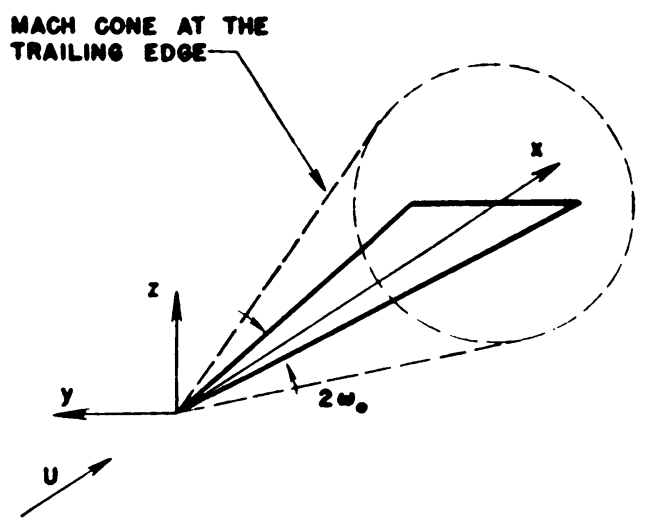

FIG. 1. Delta wing in a supersonic flow. are constant along each radial line emanating from the given origin. The concept of a conical flow was given by Busemann ${ }^{4}$ who developed certain general techniques for treating these flows and who applied the method to several problems including Schlichting's problem.

The methods of analysis used by Busemann have, however, proved to be rather obscure, and it has been found difficult to follow these methods in the solution of additional conical flow problems, in particular the currently very interesting problem of the lift of a delta wing. A new method of treating these conical flow airfoil problems which uses the well known theory of conformal transformation has been devised. It is the purpose of the present paper to discuss this method and to apply this method to the problem of the lift of a delta wing. In this application it is only necessary to consider the case for which the leading edges of the delta wing are within the Mach cone from the vertex. The other case for which the leading edges are outside the Mach cone has already been solved by Puckett.

In the present method no essential mathematical difference is found in the solution of the two cases.

* Received May 21, 1946.

1 J. Ackeret, Z.F.M., 16, 72 (1925).

? H. Schlichting, Luftfahrtforschung, 13, 320 (1936).

${ }^{3}$ A. J. Puckett, Aero. Sci. (To be published shortly).

4. Busemann, Schriften der Deutschen Akademie für Luftfahrtforschung, 7B, 105 (1943). Also Luftfahrtforschung, 12, 210 (1935). 
2. General theory of conical flows. It is well known that the linearized theory of steady supersonic flow's is based on the Prandtl-Glauert equation,

$$
\left(1-M^{2}\right) \frac{\partial^{2} P}{\partial x^{2}}+\frac{\partial^{2} P}{\partial y^{2}}+\frac{\partial^{2} P}{\partial z^{2}}=0,
$$

where the undisturbed flow of Mach number $M$ is taken to be parallel to the $x$ axis. Here, $P$ may denote a velocity or acceleration potential, or one of the velocity components $u, v, w$ in rectangular Cartesian coordinates $x, y, z$, or a property of the state of this fluid such as pressure or enthalpy. It can be seen that the coordinate transformation

$$
\left.\begin{array}{l}
r=\left[\frac{x^{2}}{M^{2}-1}-\left(y^{2}+z^{2}\right)\right]^{1 / 2}=\frac{R \cos \omega}{\mu \sqrt{M^{2}-1}}, \\
\mu=\left[1-\left(M^{2}-1\right) \frac{y^{2}+z^{2}}{x^{2}}\right]^{-1 / 2}=\left[1-\left(M^{2}-1\right) \tan ^{2} \omega\right]^{-1 / 2}, \\
\theta=\tan ^{-1}(y / z),
\end{array}\right\}
$$

where $R=\left(x^{2}+y^{2}+z^{2}\right)^{1 / 2}$ and $\omega=\tan ^{-1}\left[\left(y^{2}+z^{2}\right)^{1 / 2} / x\right]$, transforms the PrandtI-Gla uert equation, Eq. (1), into

$$
r^{2} \frac{\partial^{2} P}{\partial r^{2}}+2 r \frac{\partial P}{\partial r}+\frac{\partial}{\partial \mu}\left[\left(1-\mu^{2}\right) \frac{\partial P}{\partial \mu}\right]+\frac{.1}{1-\mu^{2}} \frac{\partial^{2} P}{\partial \theta^{2}}=0 .
$$

The surfaces on which $\theta$ is constant are the meridional planes through the $x$ axis; the surfaces on which $\mu$ is constant are cones about the $x$ axis; and the surfaces on which $r$ is constant are hyperboloids. It may also be noted that $r=0$ and $\mu=\infty$ on the Mach cone through the origin. Both $\mu$ and $r$ are real within the Mach cone and complex outside it. The harmonic solutions of Eq. (3) may be written in the form

$$
P=\sum_{m, n} A_{m n}\left\{\begin{array}{l}
r^{n} \\
r^{-n-1}
\end{array}\right\}\left\{\begin{array}{l}
P_{n}^{m}(\mu) \\
Q_{n}^{m}(\mu)
\end{array}\right\}\left\{\begin{array}{c}
\cos (m \theta) \\
\sin (m \theta)
\end{array}\right\}
$$

by the well known theory of the Laplace equation. Here, $P_{n}^{m}$ and $Q_{n}^{m}$ denote Legendre functions of the first and second kind, respectively. By introducing the normal spherical coordinates as given in Eq. (2), Eq. (4) is seen to give the harmonic solutions of the Prandtl-Glauert equation in spherical coordinates.

Busemann's conical flows are included in the general solution of Eq. (4) as a special case. For example, if $P$ is a velocity potential, then $n=1$. On the other hand, if $P$ is one of the Cartesian velocity components $(u, v, w)$, a property of the state of the fluid such as the pressure or enthalpy, or the acceleration potential, then $n=0$. It is the latter case which is of particular interest here, for $P$ is then independent of $r$, and Eq. (3) becomes

$$
\left(\mu^{2}-1\right) \frac{\partial}{\partial \mu}\left[\left(\mu^{2}-1\right) \frac{\partial P}{\partial \mu}\right]+\frac{\partial^{2} P}{\partial \theta^{2}}=0 .
$$

It is apparent that this may be reduced to the Laplace equation in two dimensions; ${ }^{*}$ in fact, if

* This result was first communicated to the author by Mr. W. D. Hayes. 


$$
s=\sqrt{\frac{\mu-1}{\mu+1}},
$$

Eq. (5) becomes

$$
s \frac{\partial}{\partial s}\left(s \frac{\partial P}{\partial s}\right)+\frac{\partial^{2} P}{\partial \theta^{2}}=0 .
$$

This is the normal form of the Laplace equation in two dimensional polar coordinates. It is seen that $s$ is a function only of $\mu$ and is thus constant on any one of the cones for which $\omega$ is constant. The relations between $s$ and $\omega$ are as follows:

$$
\begin{aligned}
& s=-\frac{\sqrt{M^{2}-1} \tan \omega}{1+\sqrt{1-\left(M^{2}-1\right) \tan ^{2} \omega}}, \\
& \sqrt{M^{2}-1} \tan \omega=\frac{2 s}{1+s^{2}} \text {. }
\end{aligned}
$$

It may further be noted that $s=1$ on the Mach cone through the origin.

Since the reduction to Eq. (6) is possible, any of the quantities which $P$ may represent can be written as the real (or imaginary) part of an analytic function of the complex variable $\zeta$ where

$$
\zeta=s e^{i \theta} .
$$

Furthermore, all the methods of treatment of such functions, in particular the method of conformal transformation, may be used in the analysis of these quantities. If $\bar{P}$ is the harmonic conjugate of $P$ and

$$
P+i \bar{F}=\mathbf{P}(\zeta),
$$

the Cauchy-Riemann equations for these conjugate functions may be written

$$
\begin{gathered}
s \frac{\partial P}{\partial s}=\frac{\partial \bar{P}}{\partial \theta}=\left(\mu^{2}-1\right) \frac{\partial P}{\partial \mu}, \\
-\frac{\partial P}{\partial \theta}=s \frac{\partial \bar{P}}{\partial s}=\left(\mu^{2}-1\right) \frac{\partial \bar{P}}{\partial \mu} .
\end{gathered}
$$

In the direct airfoil problem, the airfoil geometry is given, and if the $z$ axis is taken normal to the airfoil plane, the boundary conditions for determining the flow are thus given in terms of the disturbance velocity component $w$. It is desired in this case to compute the pressure distribution which may be easily expressed in terms of the axial disturbance velocity component $u$. In the inverse airfoil problem, a pressure distribution is defined, and it is desired that the airfoil shape be computed. In either case the boundary condition is given in terms of one velocity component and another velocity component gives the desired result. For a conical flow there are simple relations between the complex functions representing the various Cartesian velocity components. The use of these relations is the essence of the present method of treatment of conical flows. These relations between the complex functions corresponding to the Cartesian disturbance velocity components $u, v, w$ which will be written 


$$
u+i \bar{u}=\mathrm{U}(\zeta), \quad v+i \bar{v}=\mathrm{V}(\zeta), \quad w+i \bar{w}=\mathbf{W}(\zeta),
$$

are essentially the vorticity relations.

The fundamental linearized relations governing the steady flow of a fluid at supersonic speeds are the vorticity relations

$$
\frac{\partial v}{\partial z}=\frac{\partial w}{\partial y}, \quad \text { (13) } \quad \frac{\partial u}{\partial z}=\frac{\partial w}{\partial x}, \quad \text { (14) } \quad \begin{aligned}
& \partial u \\
& \partial y
\end{aligned}=\frac{\partial v}{\partial x} .
$$

and the linearized equation of continuity

$$
\frac{\partial v}{\partial y}+\frac{\partial w}{\partial z}=\left(M^{2}-1\right) \frac{\partial u}{\partial x}
$$

If these are solved simultaneously, it is easily seen that each of the velocity components obeys the Prandtl-Glauert equation, Eq. (1). For any conical flow each of the velocity components must be a function only of the coordinates $\mu$ and $\theta$. By means of this fact, Eqs. (13) to (16), respectively, may be written as follows:

$$
\begin{gathered}
\mu\left(\mu^{2}-1\right)\left(\cos \theta \frac{\partial v}{\partial \mu}-\sin \theta \frac{\partial w}{\partial \mu}\right)=\left(\cos \theta \frac{\partial w}{\partial \theta}+\sin \theta \frac{\partial v}{\partial \theta}\right) \\
-\frac{\left(\mu^{2}-1\right)^{3 / 2}}{\sqrt{M^{2}-1}} \frac{\partial w}{\partial \mu}=\mu\left(\mu^{2}-1\right) \cos \theta \frac{\partial u}{\partial \mu}-\sin \theta \frac{\partial u}{\partial \theta}, \\
-\frac{\left(\mu^{2}-1\right)^{3 / 2}}{\sqrt{M^{2}-1}} \frac{\partial v}{\partial \mu}=\mu\left(\mu^{2}-1\right) \sin \theta \frac{\partial u}{\partial \mu}+\cos \theta \frac{\partial u}{\partial \theta}, \\
-\sqrt{M^{2}=1}\left(\mu^{2}-1\right)^{3 / 2} \frac{\partial u}{\partial \mu}=\mu\left(\mu^{2}-1\right)\left(\sin \theta \frac{\partial v}{\partial \mu}+\cos \theta \frac{\partial w}{\partial \mu}\right) \\
+\left(\cos \theta \frac{\partial v}{\partial \theta}-\sin \theta \frac{\partial w}{\partial \theta}\right) .
\end{gathered}
$$

If Eqs. (18) and (19) are combined, it is seen that

$$
\begin{aligned}
& \frac{\partial u}{\partial \theta}=-\frac{\left(\mu^{2}-1\right)^{3 / 2}}{\sqrt{M^{2}-1}}\left(\cos \theta \frac{\partial v}{\partial \mu}-\sin \theta \frac{\partial w}{\partial \mu}\right), \\
& \frac{\partial u}{\partial \mu}=-\frac{\left(\mu^{2}-1\right)^{1 / 2}}{\sqrt{M^{2}-1}}\left(\sin \theta \frac{\partial v}{\partial \mu}+\cos \theta \frac{\partial w}{\partial \mu}\right) .
\end{aligned}
$$

Furthermore, Eqs. (20) and (22) show that

$$
\frac{\mu^{2}-1}{\mu}\left(\sin \theta \frac{\partial v}{\partial \mu}+\cos \theta \frac{\partial w}{\partial \mu}\right)=-\left(\cos \theta \frac{\partial v}{\partial \theta}-\sin \theta \frac{\partial w}{\partial \theta}\right) .
$$

If the derivatives with respect to $\mu$ are eliminated from Eqs. (17) and (23) by means of the Cauchy-Riemann equations [cf. Eq. (11)] for $v, w, \bar{v}$ and $\bar{w}$, these equations may be written as follows: 


$$
\begin{aligned}
& \frac{1}{\mu}\left(\sin \theta \frac{\partial v}{\partial \theta}+\cos \theta \frac{\partial w}{\partial \theta}\right)-\left(\cos \theta \frac{\partial \bar{v}}{\partial \theta}-\sin \theta \frac{\partial \bar{w}}{\partial \theta}\right)=0, \\
& \frac{1}{\mu}\left(\sin \theta \frac{\partial \bar{v}}{\partial \theta}+\cos \theta \frac{\partial \bar{w}}{\partial \theta}\right)+\left(\cos \theta \frac{\partial v}{\partial \theta}-\sin \theta \frac{\partial w}{\partial \theta}\right)=0 .
\end{aligned}
$$

If Eq. (25) is multiplied by $i$ and added to Eq. (24), it is seen that

$$
\frac{1}{\mu}\left(\sin \theta \frac{\partial \mathrm{V}}{\partial \theta}+\cos \theta \frac{\partial \mathrm{W}}{\partial \theta}\right)+i\left(\cos \theta \frac{\partial \mathrm{V}}{\partial \theta}-\sin \theta \frac{\partial \mathrm{W}}{\partial \theta}\right)=0 \text {. }
$$

Since $\mathbf{V}$ and $\mathbf{W}$ are functions of the complex variable $\zeta$, this may further be written

$$
\frac{d \mathrm{~V}}{d \zeta}=\frac{d \mathrm{~W}}{d \zeta} \frac{i \mu \sin \theta-\cos \theta}{\sin \theta+i \mu \cos \theta}
$$

and, by the definition of $s$ and Eq. (9),

$$
\frac{d V}{d \zeta}=i \frac{1-\zeta^{2}}{1+\zeta^{2}} \frac{d W}{d \zeta}
$$

A similar treatment of Eqs. (21) and (22), V being eliminated by Eqs. (17) and (27), shows that

$$
\frac{d \mathrm{U}}{d \zeta}=-\frac{2 \zeta}{\left(1+\zeta^{2}\right) \sqrt{M^{2}-1}} \frac{d \mathrm{~W}}{d \zeta} .
$$

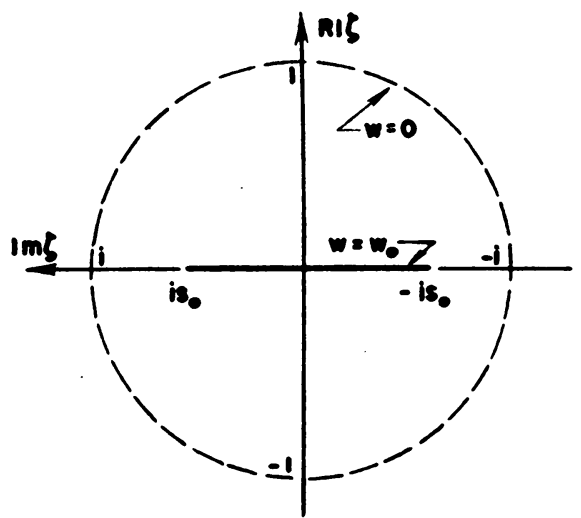

Fig. 2. Boundary conditions in the $\zeta$ plane.

These two relations, Eqs. (28) and (29), are the fundamental relations for the present treatment of conical supersonic flow problems.

3. Example. Lift of a delta wing. The general techniques developed in the previous section will now be used to compute the lift of a delta wing at a small angle of attack for the case in which the leading edges are inside of the Mach cone (see Fig. 1). The $z$ axis is taken normal to the airfoil. The conditions in the $\zeta$ plane are shown in Fig. 2. Note that the airfoil cuts the $\zeta$ plane on the imaginary axis. The boundary conditions for determining the vertical velocity $w$ are then

$$
\begin{aligned}
& w=0 \text { on } s=1, \\
& w=w_{0}=-U \alpha \text { on the airfoil, }
\end{aligned}
$$

where $U$ is the velocity of the mean flow and $\alpha$ is the angle of attack of the airfoil.

This boundary value problem can be solved by conformal transformation. First, apply the transformation

$$
\zeta_{1}=-\frac{i}{2}\left(\zeta-\frac{1}{\zeta}\right)
$$


This maps the interior of the unit circle in the $\zeta$ plane into the entire $\zeta_{1}$ plane with the region $R e \zeta>0$ corresponding to the region $\operatorname{Im} \zeta_{1}>0$. This transformed plane is
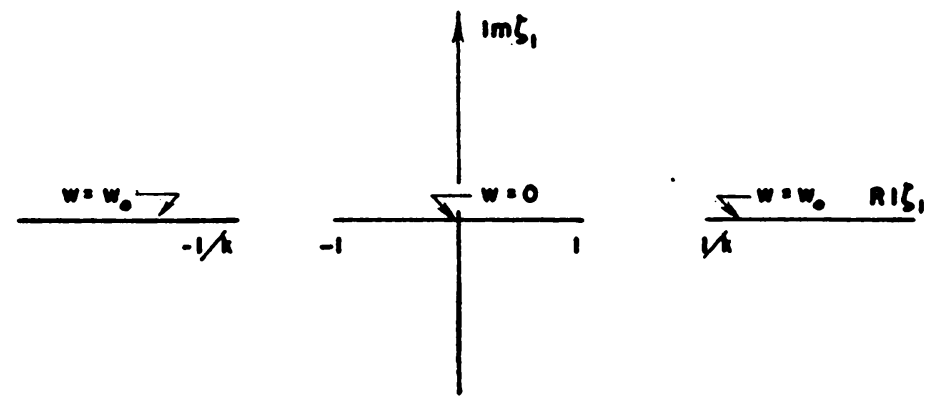

FIG. 3. Boundary conditions in the $\zeta_{1}$ plane.

shown in Fig. 3. The points at the wing tips, $\zeta= \pm i s_{0}$, are transformed into the points $\zeta_{1}= \pm 1 / k$ where

$$
k=\frac{2 s_{0}}{1+s_{0}^{2}}=\sqrt{M^{2}-1} \tan \omega_{0} .
$$

Second, apply the transformation

$$
\zeta_{2}=\int_{0}^{\zeta_{1}} \frac{d \zeta_{1}}{\sqrt{\left(1-\zeta_{1}^{2}\right)\left(1-k^{2} \zeta_{1}^{2}\right)}},
$$

i.c.,

$$
\zeta_{1}=\operatorname{sn}\left(\zeta_{2}\right) \text {, }
$$

where the elliptic function has the modulus $k$. Then the region $\operatorname{Im} \zeta_{1}>0$ is mapped into the rectangle having its corners at $\zeta_{2}= \pm K, i K^{\prime} \pm K$ where $K$ and $K^{\prime}$ are the complete elliptic integrals of the first kind having a modulus of $k$ and $k^{\prime}$ where

$$
k^{\prime}=. \sqrt{1-k^{2}}=\frac{1-s_{0}^{2}}{1+s_{0}^{2}} .
$$

By integrating around the slit from -1 to 1 in the $\zeta_{1}$ plane, it is seen that the region $\operatorname{Im} \zeta_{1}<0$ maps into the rectangle having corners at $\zeta_{2}=2 K \pm K, 2 K \pm K+i K^{\prime}$.

Now, the transformation given by Eq. (31) is double valued, i.e., two points in the $\zeta$ plane correspond to each point in the $\zeta_{1}$ plane. The $\zeta_{1}$ plane must thus be considered as a two sheeted Riemann surface with one sheet corresponding to the interior of the unit circle in the $\zeta$ plane and the other sheet corresponding to the exterior of the unit circle in the $\zeta$ plane. Furthermore, the value of the downwash velocity $w$ must be equal and opposite at inverse points in the $\zeta$ plane. This permits the analytic continuation of $w$ throughout the entire $\zeta$ plane; in particular it is seen that $w=-w_{0}$ on the exterior points corresponding to the airfoil. The two sheets in the $\zeta_{1}$ plane are connected through the slit from -1 to +1 . A contour cutting this line passes from the upper to the lower sheet or vice-versa. The sheet which corresponds to the exterior region of the $\zeta$ plane is thus seen to be mapped into the rectangle having corners at $\zeta_{2}=K \pm 2 K, K \pm 2 K-i K^{\prime}$. The entire plane is mapped into a basic rec- 
tangle in the $\zeta_{2}$ plane as shown in Fig. 4. As $\zeta_{1}$ has periorls of $4 K, 2 i K^{\prime}$ [see Eq. (34)] in $\zeta_{2}$, this pattern is repeated throughout the $\zeta_{2}$ plane.

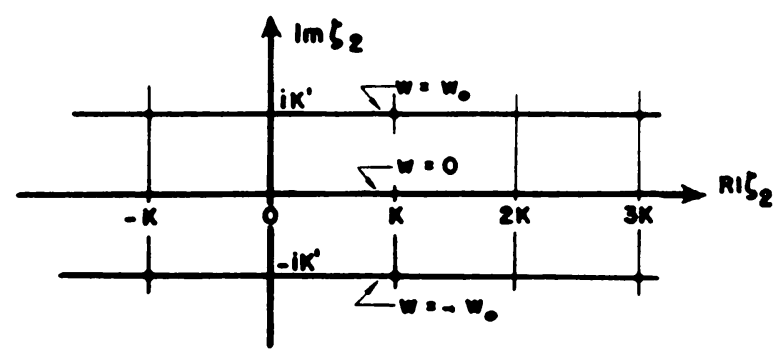

FIG. 4. Boundary conditions in the $\zeta_{2}$ plane.

The function $d \mathbf{W} / d \zeta_{2}$ (but not $\mathbf{W}$ itself) must be doubly periodic in the $\zeta_{2}$ plane with periods $4 K$ and $2 i K^{\prime}$, the first corresponding to a loop around the points $\zeta_{1}= \pm 1$ and the second corresponding to a loop around the points $\zeta_{1}=1,1 / k$ or $-1,-1 / k$. The only singularities of $\mathbf{W}$ or $d \mathrm{~W} / d \zeta_{2}$ must be at the points corresponding to the airfoil leading edges, i.e., at the points conjugate to $i K^{\prime} \pm K$. Finally $d \mathbf{W} / d \zeta_{2}$ must be pure imaginary on the lines $\operatorname{Im} \zeta_{2}=n K^{\prime}$ and $R e \zeta_{2}=K+2 n K$ ( $n$ being any integer). All of these conditions are satisfied by the Jacobian elliptic function

$$
\frac{d W}{d \zeta_{2}}=i D c d^{2 n}\left(\zeta_{2}\right)
$$

where $n$ is any positive integer and $D$ is a real constant. If this is integrated it is seen that for $n>0, W$ has a pole of order $2 n-1$ at the wing tips. The cases for $n>1$ can then be discarded as the singularity at the wing tips is seen to correspond to a sourcesink complex which has an infinite total lift. Furthermore, the case for $n=0$ may be discarded as [see Eq. (29)] it requires that $\mathbf{U}(\zeta)$ have a logarithmic singularity on the Mach cone. The appropriate solution is thus

$$
\frac{d \mathrm{~W}}{d \zeta_{2}}=i D c d^{2}\left(\zeta_{2}\right) \text {. }
$$

The constant $D$ may be evaluated from the fact that

$$
w_{0}=R l\left\{\int_{0}^{i K^{\prime}} \frac{d \mathrm{~W}}{d \zeta_{2}} d \zeta_{2}\right\} .
$$

If this integration is carried out, it is seen that

$$
D=-\frac{k^{2} w_{0}}{E\left(k^{\prime}\right)},
$$

where $E\left(k^{\prime}\right)$ is the complete elliptic integral of the second kind having a modulus $k^{\prime}$ as given by Eq. (35).

If the variable $\zeta_{2}$ is eliminated from Eq. (37) by means of Eq. (31) and (33), it is seen that 


$$
\frac{d \mathbf{W}}{d \zeta}=-\frac{2 w_{0}}{k E\left(k^{\prime}\right)} \frac{\left(1+\zeta^{2}\right)^{2}}{\left[\left(\zeta^{\prime \prime}+s_{0}^{2}\right)\left(\zeta_{2}+\frac{1}{s_{0}^{2}}\right)\right]^{3 / 2}} .
$$

Thus, from Eq. (29),

$$
\frac{d \mathbf{U}}{d \zeta}=\frac{4 w_{0}}{k E\left(k^{\prime}\right) \mathbf{V}^{\prime} M^{2}-1} \frac{\zeta\left(1+\zeta^{2}\right)}{\left[\left(\zeta^{2}+s_{0}^{2}\right)\left(\zeta^{2}+\frac{1}{s_{0}^{2}}\right)\right]^{3 / 2}}
$$

Since $U=0$ at $\zeta=1$, the integral of Eq. $(41)$ is

$$
\mathbf{U}=\cdot \frac{k w_{0}}{E\left(k^{\prime}\right) \sqrt{M^{2}-1}} \frac{\zeta^{2}-1}{\left[\left(\zeta^{2}+s_{0}^{2}\right)\left(\zeta^{2}+\frac{1}{s_{0}^{2}}\right)\right]^{1 / 2}} .
$$

On the top side of the airfoil $\zeta=i \eta$ wher $-s_{0}<\eta<s_{0}$, so

$$
u=-\underset{E\left(k^{\prime}\right) \sqrt{M^{2}}-\overline{1}}{\frac{k w_{\theta}}{\left[\left(s_{0}^{2}-\eta^{2}\right)\left(\frac{1}{s_{0}^{2}}-\eta^{2}\right)\right]^{1 / 2}}}
$$

This result may be considerably simplified if we introduce [from Eq. (8) and (30)]

$$
\begin{aligned}
k & =\sqrt{M^{2}-1} \tan \omega_{\theta} \\
w_{0} & =-U \alpha
\end{aligned}
$$

and

$$
t=\frac{\tan \omega}{\tan \omega_{0}}
$$

Equation (43) then becomes

$$
\frac{4}{\alpha}\left(\frac{u}{U}\right)=\frac{4 \tan \omega_{0}}{E\left(k^{\prime}\right) \sqrt{1-t^{2}}} .
$$

The slope of the lift curve $d C_{L} / d \alpha$ is given by the mean value of $4 / \alpha(u / U)$ over the surface of the wing; thus

$$
\frac{d C_{L}}{d \alpha}=\frac{4}{E\left(k^{\prime}\right)} \int_{0}^{\omega_{0}} \frac{\sec ^{2} \omega d \omega}{\sqrt{1-t^{2}}}
$$

and, by Eq. (44),

$$
\frac{d C_{L}}{d \alpha}=\frac{2 \pi \tan \omega_{0}}{E\left(k^{\prime}\right)}
$$

In the limit for which $\omega_{\theta}$ or $s_{0} \rightarrow 0, k^{\prime} \rightarrow 1$; so $E\left(k^{\prime}\right) \rightarrow 1$. For this case which was given by Jones ${ }^{5}$

- R. T. Jones, N.A.C.A., Technical Note 1032 (1945). 


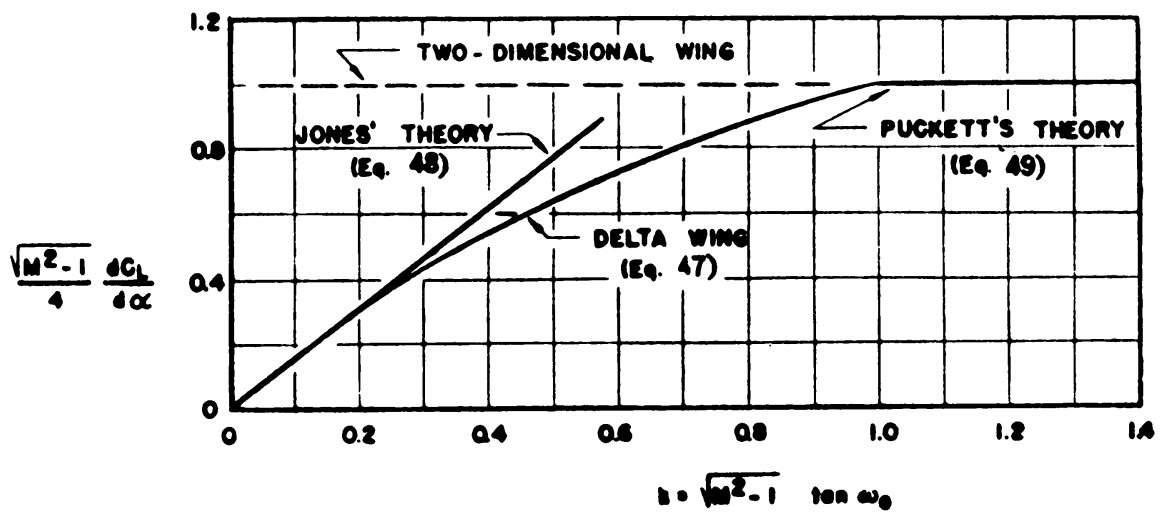

Fig. 5. Lift of a delta wing.

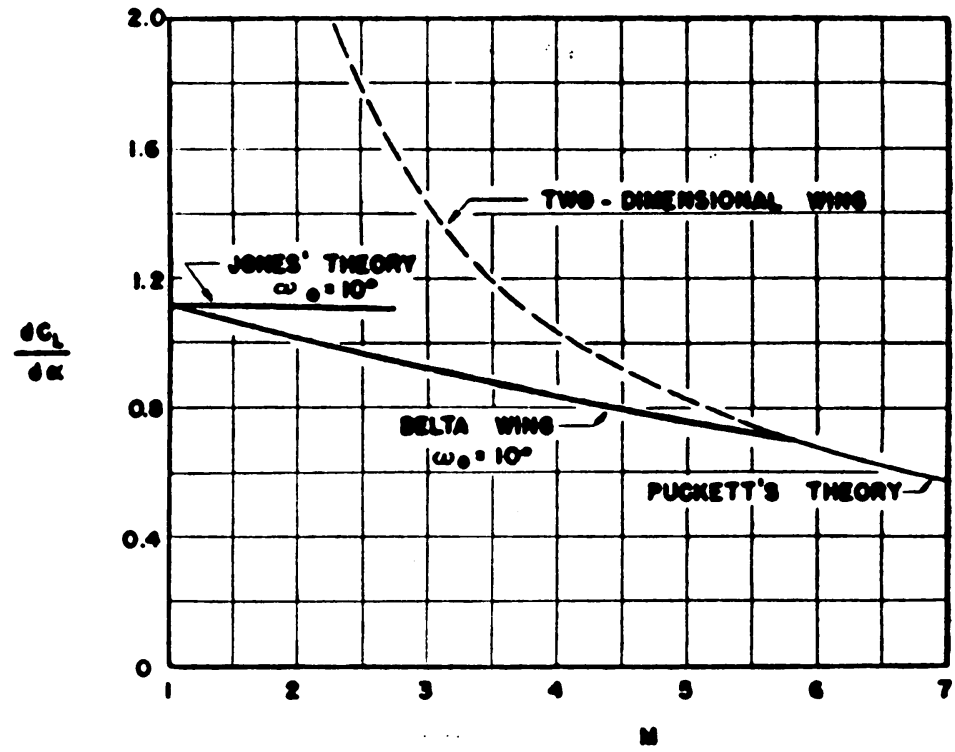

FIG. 6. $d C_{L} / d \alpha$ vs. $M$ for a delta wing with $\omega=10^{\circ}$.

$$
\frac{d C_{L}}{d \alpha}=2 \pi \tan \omega_{0} .
$$

On the other limit for which $s_{0} \rightarrow 1, k^{\prime} \rightarrow 0$; so $E\left(k^{\prime}\right) \rightarrow \pi / 2$. For this case

$$
\frac{d C_{L}}{d \alpha}=4 \tan \omega_{0}=\frac{4}{\sqrt{M^{2}-1}} .
$$

This limit, the same as the two dimensional solution, had previously been obtained by Puckett.

It may further be noted that the quantity $\frac{1}{2} \sqrt{M^{2}-1} d C_{L} / d \alpha$ is a function only of the parameter $k=\sqrt{M^{2}-1} \tan \omega_{0}$. This result is shown graphically in Fig. 5, and the slope of the lift curve for a particular case, $\omega_{0}=10^{\circ}$, is shown as a function of Mach number in Fig. 6. 